\title{
Application of Lagrange equations to 2D double spring- pendulum in generalized coordinates
}

\author{
N.O. Nenuwe \\ Department of Physics, Federal University of Petroleum Resources, P. M. B. 1221, \\ Effurun, Delta State, Nigeria, Nigeria \\ Correspondence: nenuwe.nelson@fupre.edu.ng (i) https://orcid.org/0000-0002-3112-3869
}

Received: $31^{\text {st }}$ December 2018; Revised: $30^{\text {th }}$ July 2019; Accepted: $31^{\text {st }}$ December 2019

\begin{abstract}
In this study, the Lagrange's equations of motion for a 2D double spring-pendulum with a time dependent spring extension have been derived and solved approximately. The resulting equations are also solved numerically using Maple, and plots of motion for the pendulum bobs $\mathrm{ml}$ and $m 2$ are presented and compared. It was observed that motion along the $\mathrm{x}$-axis is characterized by sine wave function while motion along $\mathrm{y}$-axis is characterized by cosine wave function with slightly changing amplitudes. Change in stiffness constant, angle of deflection, mass of pendulum bob and spring length were found to have significant effect on the dynamics of the double spring-pendulum. The periodic and chaotic behaviour noticed in this study is consistent with current literature on spring-pendulum systems.
\end{abstract}

Keywords: Lagrange equations, double spring-pendulum.

\section{Introduction}

The two dimensional (2D) double pendulum is a typical example of chaotic motion in classical mechanics. The pattern of its motion is well known to change drastically as the energy is increased from zero to infinity (Biglari and Jami 2016). However, at low and very high energies the system represents coupled harmonic oscillators, and can be considered as an integrable system. But, at intermediate energies, the system is known to exhibit chaotic features.

Double spring-pendulum is a classical mechanical system consisting of two bobs of mass $m_{1}$ and $m_{2}$ fixed to the ends of two weightless elastic springs with stiffness constants $k_{1}$ and $k_{2}$, and the angle of deflections $q_{1}$ and $q_{2}$, respectively. The second spring is connected to the first mass as shown in Fig. 1.

The dynamics of double spring-pendulum appears to be scanty in literature, though there are reports on single and double spring pendulum systems. Marcus et al. (2016) studied the order-chaos-order transition of 
spring pendulum using the Hamiltonian formulation. Numerical analysis of the equations of motion for double pendulum was reported by Smith (2002) using Maple soft. de Sousa et al. (2017) reported on the energy distribution in spring pendulum. Lewin et al. (2015) numerically analyzed the dynamics of single and double pendulum using MATLAB. Also, double pendulum numerical analysis with Lagrangian and Hamiltonian equations of motions using MATLAB was reported by Biglari and Jami (2016). Despite these studies, no reports to the best of my knowledge have been made on double spring-pendulum with time dependent extension in spring length using Lagrangian formulations.

Also, it is well known that physical systems can be described by their Lagrangian, and from the Lagrangian function one can obtain second order differential equations of motion describing such dynamic systems. In most cases the exact solution cannot be obtained for these Lagrange equations of motion, and this leads to employing alternative numerical approach to solve such equations (Baleanu et al. 2015).

The interest in this study is to analytically obtain the equations of motion for a double spring-pendulum with time dependent spring-extension. Hence, Lagrangian formulation of mechanics is used to derive the equations of motion for the system. The resulting Lagrange's equations are solved approximately and numerically using MAPLE software.

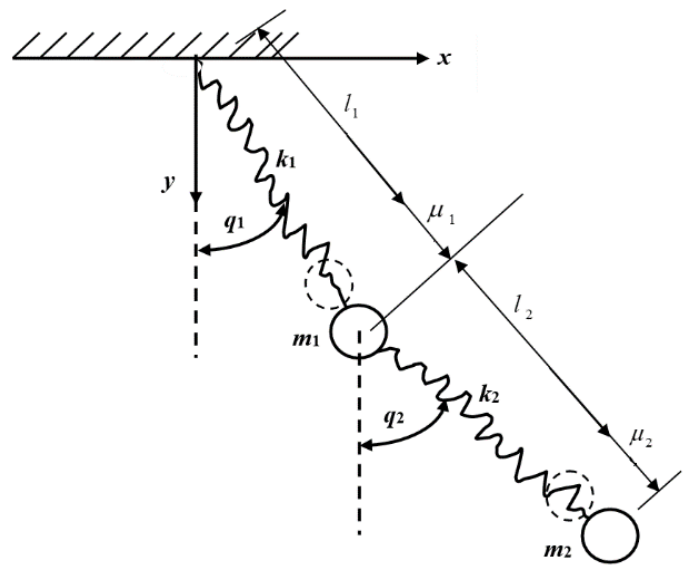

Fig. 1. Schematic diagram of a double spring-pendulum

\section{Derivation of the Lagrange's equations of motion}

Suppose the positions of mass $m_{1}$ and $m_{2}$ at any time in space is expressed in Cartesian coordinates as $\left(x_{1}, y_{1}\right)$ and $\left(x_{2}, y_{2}\right)$, unstretched lengths of the 
springs are $l_{1}$ and $l_{2}$, and the springs extend by $\mu_{1}(\mathrm{t})$ and $\mu_{2}(\mathrm{t})$ when the respective masses are attached as shown in Figure 1.

At the point of suspension, the positions of the bobs are given by the following equations:

$$
\begin{aligned}
& x_{1}(t)=\left(l_{1}+\mu_{1}(t)\right) \sin q_{1}(t) \\
& y_{1}(t)=-\left(l_{1}+\mu_{1}(t)\right) \cos q_{1}(t) \\
& x_{2}(t)=\left(l_{1}+\mu_{1}(t)\right) \sin q_{1}(t)+\left(l_{2}+\mu_{2}(t)\right) \sin q_{2}(t) \\
& y_{2}(t)=-\left(l_{1}+\mu_{1}(t)\right) \cos q_{1}(t)-\left(l_{2}+\mu_{2}(t)\right) \cos q_{2}(t)
\end{aligned}
$$

The total kinetic energy $(\mathrm{T})$ of the system is given by:

$$
\begin{aligned}
& T=\frac{1}{2} m_{1}\left(\dot{x}_{1}^{2}(t)+\dot{y}_{1}^{2}(t)\right)+\frac{1}{2} m_{2}\left(\dot{x}_{2}^{2}(t)+\dot{y}_{2}^{2}(t)\right) \\
& T(q, \mu, \dot{q}, \dot{\mu})=\frac{1}{2} m_{1}\left\{\dot{\mu}_{1}^{2}(t)+\left(l_{1}+\mu_{1}(t)\right)^{2} \dot{q}_{1}^{2}(t)\right\}+ \\
& \quad \frac{1}{2} m_{2}\left\{\begin{array}{l}
\dot{\mu}_{1}^{2}(t)+\dot{\mu}_{2}^{2}(t)+\left(l_{1}+\mu_{1}(t)\right)^{2} \dot{q}_{1}^{2}(t)+\left(l_{2}+\mu_{2}(t)\right)^{2} \dot{q}_{2}^{2}(t) \\
+\left(2 \dot{\mu}_{1}(t) \dot{\mu}_{2}(t)+2\left(l_{1}+\mu_{1}(t)\right)\left(l_{2}+\mu_{2}(t) \dot{q}_{1}(t) \dot{q}_{2}(t)\right) \cos \left(q_{1}-q_{2}\right)\right. \\
+\left(2 \left(l_{2}+\mu_{2}(t) \dot{\mu}_{1}(t) \dot{q}_{2}(t)-2\left(l_{1}+\mu_{1}(t) \dot{\mu}_{2}(t) \dot{q}_{1}(t)\right) \sin \left(q_{1}-q_{2}\right)\right.\right.
\end{array}\right\}
\end{aligned}
$$

By taking a plane at distance $\left(l_{l}+l_{2}\right)$ below the point of suspension of Figure 1 as a reference level, the potential $(V)$ energy of the system is then given by:

$$
\begin{aligned}
V(q, \mu)= & m_{1} g\left\{l_{1}+l_{2}-\left(l_{1}+\mu_{1}(t)\right) \cos q_{1}(t)\right\}+ \\
& m_{2} g\left\{l_{1}+l_{2}-\left(l_{1}+\mu_{1}(t)\right) \cos q_{1}(t)-\left(l_{2}+\mu_{2}(t)\right) \cos q_{2}(t)\right\}+ \\
& \frac{1}{2} k_{1} \mu_{1}^{2}(t)+\frac{1}{2} k_{2} \mu_{2}^{2}(t)
\end{aligned}
$$

From this, the Lagrangian function for the system is given by:

$$
\begin{aligned}
& L=T-V \\
& \qquad \begin{aligned}
L(q, \mu, \dot{\mu}, \dot{q})= & \frac{1}{2} m_{1}\left\{\dot{\mu}_{1}^{2}(t)+\left(l_{1}+\mu_{1}(t)\right)^{2} \dot{q}_{1}^{2}(t)\right\}+\frac{1}{2} m_{2}\left\{\dot{\mu}_{1}^{2}(t)+\dot{\mu}_{2}^{2}(t)+\left(l_{1}+\mu_{1}(t)\right)^{2} \dot{q}_{1}^{2}(t)\right. \\
& +\left(l_{2}+\mu_{2}(t)\right)^{2} \dot{q}_{2}^{2}(t)+\left(2 \dot{\mu}_{1}(t) \dot{\mu}_{2}(t)+2\left(l_{1}+\mu_{1}(t)\right)\left(l_{2}+\mu_{2}(t) \dot{q}_{1}(t) \dot{q}_{2}(t)\right) \cos \left(q_{1}-q_{2}\right)\right. \\
& +\left(2\left(l_{2}+\mu_{2}(t) \dot{\mu}_{1}(t) \dot{q}_{2}(t)-2\left(l_{1}+\mu_{1}(t) \dot{\mu}_{2}(t) \dot{q}_{1}(t)\right) \sin \left(q_{1}-q_{2}\right)\right\}\right. \\
& -m_{1} g\left\{l_{1}+l_{2}-\left(l_{1}+\mu_{1}(t)\right) \cos q_{1}(t)\right\}-m_{2} g\left\{l_{1}+l_{2}-\left(l_{1}+\mu_{1}(t)\right) \cos q_{1}(t)\right. \\
& \left.-\left(l_{2}+\mu_{2}(t)\right) \cos q_{2}(t)\right\}+\frac{1}{2} k_{1} \mu_{1}^{2}(t)+\frac{1}{2} k_{2} \mu_{2}^{2}(t)
\end{aligned}
\end{aligned}
$$


The Lagrange's equations (Murray 1967, Goldstein et al. 2000, Martin and Salomonson 2009) associated with the generalized coordinates $q_{1}(t), q_{2}(t)$, $\mu_{1}(t)$, and $\mu_{2}(t)$ are given by:

$$
\begin{array}{ll}
\frac{d}{d t}\left(\frac{\partial L}{\partial \dot{q}_{1}}\right)-\left(\frac{\partial L}{\partial q_{1}}\right)=0, & \frac{d}{d t}\left(\frac{\partial L}{\partial \dot{q}_{2}}\right)-\left(\frac{\partial L}{\partial q_{2}}\right)=0, \\
\frac{d}{d t}\left(\frac{\partial L}{\partial \dot{\mu}_{1}}\right)-\left(\frac{\partial L}{\partial \mu_{1}}\right)=0, & \frac{d}{d t}\left(\frac{\partial L}{\partial \dot{\mu}_{2}}\right)-\left(\frac{\partial L}{\partial \mu_{2}}\right)=0 .
\end{array}
$$

Now differentiating equation (8) accordingly, and substituting into equation (9) gives four Lagrange's equations of motion for the system; one equation for each degree of freedom (i.e., $q_{1}, q_{2}, \mu_{1}$ and $\mu_{2}$ ). They are as follows:

$$
\begin{gathered}
\left(m_{1}+m_{2}\right)\left(l_{1}+\mu_{1}(t)\right) \ddot{q}_{1}(t)+m_{2}\left(l_{2}+\mu_{2}(t)\right) \cos \left(q_{1}-q_{2}\right) \ddot{q}_{2}(t)-m_{2} \sin \left(q_{1}-q_{2}\right) \ddot{\mu}_{2}(t) \\
=-2\left(m_{1}+m_{2}\right)\left(l_{1}+\mu_{1}(t)\right) \dot{\mu}_{1}(t) \dot{q}_{1}(t)-m_{2}\left(l_{2}+\mu_{2}(t)\right) \sin \left(q_{1}-q_{2}\right) \dot{q}_{1}^{2}(t) \\
\quad-2 m_{2} \cos \left(q_{1}-q_{2}\right) \dot{\mu}_{2}(t) \dot{q}_{2}(t)-\left(m_{1}+m_{2}\right) g \sin q_{1} \\
m_{2}\left(l_{2}+\mu_{2}(t)\right) \ddot{q}_{2}(t)+m_{2} \sin \left(q_{1}-q_{2}\right) \ddot{\mu}_{1}(t) \\
=-2 m_{2} \cos \left(q_{1}-q_{2}\right) \dot{\mu}_{1}(t) \dot{q}_{1}(t)+m_{2}\left(l_{1}+\mu_{1}(t) \sin \left(q_{1}-q_{2}\right) \dot{q}_{1}^{2}(t)\right. \\
\quad-2 m_{2}\left(l_{2}+\mu_{2}(t)\right) \dot{\mu}_{2}(t) \dot{q}_{2}(t)-m_{2} g \sin q_{2} \\
m_{2}\left(l_{2}+\mu_{2}(t)\right) \sin \left(q_{1}-q_{2}\right) \ddot{q}_{2}(t)+\left(m_{1}+m_{2}\right) \ddot{\mu}_{1}(t)+m_{2} \cos \left(q_{1}-q_{2}\right) \ddot{\mu}_{2}(t) \\
=-2 m_{2} \sin \left(q_{1}-q_{2}\right) \dot{\mu}_{2}(t) \dot{q}_{2}(t)+\left(m_{1}+m_{2}\right)\left(l_{1}+\mu_{1}(t)\right) \dot{q}_{1}^{2}(t) \\
\quad+m_{2}\left(l_{2}+\mu_{2}(t)\right)\left(1+\cos \left(q_{1}-q_{2}\right)\right) \dot{q}_{2}^{2}(t)+m_{1} g\left(\cos q_{1}+\cos q_{2}\right)-k_{1} \mu_{1}(t) \\
-m_{2}\left(l_{1}+\mu_{1}(t)\right) \sin \left(q_{1}-q_{2}\right) \ddot{q}_{1}(t)+m_{2} \cos \left(q_{1}-q_{2}\right) \ddot{\mu}_{1}(t)+m_{2} \ddot{\mu}_{2}(t) \\
=2 m_{2} \sin \left(q_{1}-q_{2}\right) \dot{\mu}_{1}(t) \dot{q}_{1}(t)-m_{2}\left(\left(l_{2}+\mu_{2}(t)\right)-\left(l_{1}+\mu_{1}(t)\right) \cos \left(q_{1}-q_{2}\right)\right) \dot{q}_{1}^{2}(t) \\
\quad+m_{2} g \cos q_{2}-k_{2} \mu_{2}(t)
\end{gathered}
$$

Equations (10) - (13) represent a pair of coupled second order differential equations describing the unconstrained motion of a double spring-pendulum.

Generally, equations of motion can be represented in matrix form as:

$$
M \ddot{p}(t)+c_{1} \dot{p}(t)+c_{2} p(t)=f(t)
$$

Where, $c_{1}$ and $c_{2}$ are the damping coefficient and stiffness matrices. Rearranging equation (14), one obtains the mass matrix $\mathrm{M}$ and rest matrix $\mathrm{R}$ in the representation given by equation (15):

$$
M \ddot{p}(t)=R(t)
$$


where:

$$
\ddot{p}(t)=M^{-1} R(t)
$$

and

$$
\ddot{p}(t)=\left(\begin{array}{c}
\ddot{q}_{1} \\
\ddot{q}_{2} \\
\ddot{\mu}_{1} \\
\ddot{\mu}_{2}
\end{array}\right)
$$

Substituting equations (10), (11), (12) and (13) into (15), we obtain

$$
\begin{gathered}
M=\left(\begin{array}{cccc}
\left(m_{1}+m_{2}\right)\left(l_{1}+\mu_{1}(t)\right) & m_{2}\left(l_{2}+\mu_{2}(t)\right) \cos \left(q_{1}-q_{2}\right) & 0 & -m_{2} \sin \left(q_{1}-q_{2}\right) \\
0 & m_{2}\left(l_{2}+\mu_{2}(t)\right) & m_{2} \sin \left(q_{1}-q_{2}\right) & 0 \\
0 & m_{2}\left(l_{2}+\mu_{2}(t)\right) \sin \left(q_{1}-q_{2}\right) & \left(m_{1}+m_{2}\right) & m_{2} \cos \left(q_{1}-q_{2}\right) \\
-m_{2}\left(l_{1}+\mu_{1}(t)\right) \sin \left(q_{1}-q_{2}\right) & 0 & m_{2} \cos \left(q_{1}-q_{2}\right) & m_{2}
\end{array}\right) \\
\ddot{p}(t)=\left(\begin{array}{c}
\ddot{q}_{1} \\
\ddot{q}_{2} \\
\ddot{\mu}_{1} \\
\ddot{\mu}_{2}
\end{array}\right)
\end{gathered}
$$

$$
R=\left(\begin{array}{c}
-2\left(m_{1}+m_{2}\right)\left(l_{1}+\mu_{1}(t)\right) \dot{\mu}_{1}(t) \dot{q}_{1}(t)-m_{2}\left(l_{2}+\mu_{2}(t)\right) \sin \left(q_{1}-q_{2}\right) \dot{q}_{1}^{2}(t)-2 m_{2} \cos \left(q_{1}-q_{2}\right) \dot{\mu}_{2}(t) \dot{q}_{2}(t) \\
-\left(m_{1}+m_{2}\right) g \sin q_{1} \\
-2 m_{2} \cos \left(q_{1}-q_{2}\right) \dot{\mu}_{1}(t) \dot{q}_{1}(t)+m_{2}\left(l_{1}+\mu_{1}(t) \sin \left(q_{1}-q_{2}\right) \dot{q}_{1}^{2}(t)-2 m_{2}\left(l_{2}+\mu_{2}(t)\right) \dot{\mu}_{2}(t) \dot{q}_{2}(t)\right. \\
-m_{2} g \sin q_{2} \\
-2 m_{2} \sin \left(q_{1}-q_{2}\right) \dot{\mu}_{2}(t) \dot{q}_{2}(t)+\left(m_{1}+m_{2}\right)\left(l_{1}+\mu_{1}(t)\right) \dot{q}_{1}^{2}(t)+m_{2}\left(l_{2}+\mu_{2}(t)\right)\left(1+\cos \left(q_{1}-q_{2}\right)\right) \dot{q}_{2}^{2}(t) \\
+m_{1} g\left(\cos q_{1}+\cos q_{2}\right)-k_{1} \mu_{1}(t) \\
2 m_{2} \sin \left(q_{1}-q_{2}\right) \dot{\mu}_{1}(t) \dot{q}_{1}(t)-m_{2}\left(\left(l_{2}+\mu_{2}(t)\right)-\left(l_{1}+\mu_{1}(t)\right) \cos \left(q_{1}-q_{2}\right)\right) \dot{q}_{1}^{2}(t)+m_{2} g \cos q_{2}-k_{2} \mu_{2}(t)
\end{array}\right)
$$

The matrix equations in (18), (19) and (20) represent a four dimensional system of equations of motion for the double spring-pendulum in generalized coordinates: $q_{1}, q_{2}, \mu_{1}$ and $\mu_{2}$. The size of the mass matrix $M$ is $4 \times 4$ and that of the rest matrix $R$ is $4 \times 1$.

The coupled second order differential equations can only be solved approximately (Murray, 1967). Considering a case where 
$m_{1}=m_{2}=m, \mu_{1}=\mu_{2}=\mu, l_{1}=l_{2}=l$ and $k_{1}=k_{2}$, these equations simplifies to the following:

$$
\begin{aligned}
& 2(l+\mu(t)) \ddot{q}_{1}(t)+(l+\mu(t)) \cos \left(q_{1}-q_{2}\right) \ddot{q}_{2}(t)-\sin \left(q_{1}-q_{2}\right) \ddot{\mu}_{2}(t) \\
& =-4(l+\mu(t)) \dot{\mu}_{1}(t) \dot{q}_{1}(t)-\left(l_{2}+\mu_{2}(t)\right) \sin \left(q_{1}-q_{2}\right) \dot{q}_{1}^{2}(t)-2 \cos \left(q_{1}-q_{2}\right) \dot{\mu}_{2}(t) \dot{q}_{2}(t)-2 g \sin q_{1} \\
& (l+\mu(t)) \ddot{q}_{2}(t)+\sin \left(q_{1}-q_{2}\right) \ddot{\mu}_{1}(t) \\
& =-2 \cos \left(q_{1}-q_{2}\right) \dot{\mu}_{1}(t) \dot{q}_{1}(t)+\left(l+\mu(t) \sin \left(q_{1}-q_{2}\right) \dot{q}_{1}^{2}(t)-2(l+\mu(t)) \dot{\mu}_{2}(t) \dot{q}_{2}(t)-g \sin q_{2}\right. \\
& (l+\mu(t)) \sin \left(q_{1}-q_{2}\right) \ddot{q}_{2}(t)+2 \ddot{\mu}_{1}(t)+\cos \left(q_{1}-q_{2}\right) \ddot{\mu}_{2}(t)+2 \sin \left(q_{1}-q_{2}\right) \dot{\mu}_{2}(t) \dot{q}_{2}(t) \\
& \quad=2(l+\mu(t)) \dot{q}_{1}^{2}(t)+(l+\mu(t))\left(1+\cos \left(q_{1}-q_{2}\right)\right) \dot{q}_{2}^{2}(t)+g\left(\cos q_{1}+\cos q_{2}\right)-\frac{k}{m} \mu(t) \\
& -(l+\mu(t)) \sin \left(q_{1}-q_{2}\right) \ddot{q}_{1}(t)+\cos \left(q_{1}-q_{2}\right) \ddot{\mu}_{1}(t)+\ddot{\mu}_{2}(t) \\
& =2 \sin \left(q_{1}-q_{2}\right) \dot{\mu}_{1}(t) \dot{q}_{1}(t)-\left((l+\mu(t))-(l+\mu(t)) \cos \left(q_{1}-q_{2}\right)\right) \dot{q}_{1}^{2}(t)+g \cos q_{2}-\frac{k}{m} \mu(t)
\end{aligned}
$$

These equations can be solved exactly for small angle of deflection. For small oscillations, we use the approximations:

$\sin \left(q_{1}-q_{2}\right) \approx q_{1}-q_{2}, \cos \left(q_{1}-q_{2}\right) \approx 1$, and neglecting terms involving $\dot{\mu} \dot{q}, \dot{q}^{2}$, in equations (21) - (24), we get

$$
\begin{aligned}
& 2(l+\mu(t)) \ddot{q}_{1}(t)+(l+\mu(t)) \ddot{q}_{2}(t)-\left(q_{1}-q_{2}\right) \ddot{\mu}_{2}(t)=-2 g q_{1} \\
& (l+\mu(t)) \ddot{q}_{2}(t)+\left(q_{1}-q_{2}\right) \ddot{\mu}_{1}(t)=-g q_{2} \\
& (l+\mu(t))\left(q_{1}-q_{2}\right) \ddot{q}_{2}(t)+2 \ddot{\mu}_{1}(t)+\ddot{\mu}_{2}(t)=2 g-\frac{k}{m} \mu(t) \\
& -(l+\mu(t))\left(q_{1}-q_{2}\right) \ddot{q}_{1}(t)+\ddot{\mu}_{1}(t)+\ddot{\mu}_{2}(t)=g-\frac{k}{m} \mu(t)
\end{aligned}
$$

From equations (25) and (26), we have that:

$$
\begin{aligned}
& \ddot{\mu}_{1}(t)=\frac{-1}{\left(q_{1}-q_{2}\right)}\left\{(l+\mu(t)) \ddot{q}_{2}(t)+g q_{2}\right\} \\
& \ddot{\mu}_{2}(t)=\frac{1}{\left(q_{1}-q_{2}\right)}\left\{2(l+\mu(t)) \ddot{q}_{1}(t)+(l+\mu(t)) \ddot{q}_{2}(t)+2 g q_{1}\right\}
\end{aligned}
$$

Substituting equation (29) into equations (27) and (28), and neglecting terms involving $q_{1} q_{2}, q^{2},\left(q_{1}-q_{2}\right)^{2}$, we get 
$2(l+\mu(t)) \ddot{q}_{1}(t)-(l+\mu(t)) \ddot{q}_{2}(t)=-\frac{k}{m} \mu(t)\left(q_{1}-q_{2}\right)$

$2(l+\mu(t)) \ddot{q}_{1}(t)=-g q_{1}-\frac{k}{m} \mu(t)\left(q_{1}-q_{2}\right)$

The normal frequencies and normal modes that correspond to small oscillations for the double spring-pendulum is calculated from equations (30) and (31) by using equation (32)

$q_{1}=A_{1} e^{i \omega t}, \quad q_{2}=A_{2} e^{i \omega t}$.

Substituting the derivatives of (32) into (30) and (31), and simplifying gives

$$
\begin{aligned}
& \left(2(l+\mu(t)) \omega^{2}-\frac{k}{m} \mu(t)\right) A_{1}-\left((l+\mu(t)) \omega^{2}-\frac{k}{m} \mu(t)\right) A_{2}=0 \\
& \left(2(l+\mu(t)) \omega^{2}-\left(g+\frac{k}{m} \mu(t)\right)\right) A_{1}+\frac{k}{m} \mu(t) A_{2}=0
\end{aligned}
$$

In order for $A_{1}$ and $A_{2}$ to be non-zero, we must set the determinant of the coefficients equal to zero. That is,

$$
\begin{aligned}
& \left|\begin{array}{cc}
2(l+\mu(t)) \omega^{2}-\frac{k}{m} \mu(t) & -\left((l+\mu(t)) \omega^{2}-\frac{k}{m} \mu(t)\right) \\
2(l+\mu(t)) \omega^{2}-\left(g+\frac{k}{m} \mu(t)\right) & \frac{k}{m} \mu(t)
\end{array}\right|=0 \\
& \text { or } \quad 2(l+\mu)^{2} \omega^{4}-(l+\mu)\left(g+\frac{k}{m} \mu\right) \omega^{2}+g \frac{k}{m} \mu=0 \text {. On solving this }
\end{aligned}
$$

equation, we get

$$
\omega^{2}=\frac{\left(g+\frac{k}{m} \mu\right) \pm \sqrt{\left(g+\frac{k}{m} \mu\right)^{2}-8 g \frac{k}{m} \mu}}{4(l+\mu)}
$$

Using the positive and negative parts of $\omega^{2}$ in equation (36), the normal frequencies are given by

$$
f_{1}=\frac{\omega_{1}}{2 \pi}=\frac{1}{2 \pi}\left(\frac{\left(g+\frac{k}{m} \mu\right)+\sqrt{\left(g+\frac{k}{m} \mu\right)^{2}-8 g \frac{k}{m} \mu}}{4(l+\mu)}\right)^{\frac{1}{2}} \text {, }
$$




$$
f_{2}=\frac{\omega_{2}}{2 \pi}=\frac{1}{2 \pi}\left(\frac{\left(g+\frac{k}{m} \mu\right)-\sqrt{\left(g+\frac{k}{m} \mu\right)^{2}-8 g \frac{k}{m} \mu}}{4(l+\mu)}\right)^{\frac{1}{2}}
$$

Substituting the positive part of equation (36) into equation (34) gives

$$
A_{1}=\frac{-\frac{k}{m} \mu}{-\left(g+\frac{k}{m} \mu\right)+\sqrt{\left(g+\frac{k}{m} \mu\right)^{2}-8 g \frac{k}{m} \mu}} A_{2}
$$

Equation (39) corresponds to the normal mode in which the bobs of mass $m_{1}$ and $m_{2}$ are moving in opposite directions. This is called the anti-symmetric mode.

Also, substituting the negative part of equation (36) into equation (34) gives

$$
A_{1}=\frac{\frac{k}{m} \mu}{\left(g+\frac{k}{m} \mu\right)+\sqrt{\left(g+\frac{k}{m} \mu\right)^{2}-8 g \frac{k}{m} \mu}} A_{2}
$$

This corresponds to the normal mode in which the bobs are moving in the same directions.

\section{Numerical solution}

Maple software (Maple, 2018) is used to numerically solve the set of second order differential equations given by the Lagrange's equations of motion. In order to perform numerical analysis for the system, first the position coordinates $\left(x_{1}, y_{1}, x_{2}, y_{2}\right)$ are defined. Thereafter, Maple performs the following tasks for the system:

- Calculates time derivatives $\left(\dot{x}_{1}, \dot{y}_{1}, \dot{x}_{2}, \dot{y}_{2}\right)$ of the position coordinates.

- Evaluates kinetic energy for the system.

- Evaluates potential energy for the system with reference to distance below the point of suspension.

- Obtains the Lagrangian function from the difference between kinetic and potential energies. 
- Evaluates the derivatives:

$$
\frac{\partial L}{\partial q_{1}}, \frac{\partial L}{\partial q_{2}}, \frac{\partial L}{\partial \mu_{1}}, \frac{\partial L}{\partial \mu_{2}}, \frac{\partial L}{\partial \dot{q}_{1}}, \frac{\partial L}{\partial \dot{q}_{2}}, \frac{\partial L}{\partial \dot{\mu}_{1}}, \frac{\partial L}{\partial \dot{\mu}_{2}} .
$$

- Calculates the Lagrange's equations associated with the generalized coordinates $q_{1}, q_{2}, \mu_{1}, \mu_{2}$.

- Numerically solves the Lagrange's equations associated with $q_{1}, q_{2}, \mu_{1}, \mu_{2}$ for different values of $m_{1}, m_{2}, g, k_{1}, k_{2}, L_{1}$ and $L_{2}$.

New parameters $\left(\mathrm{X}_{1}, \mathrm{Y}_{1}, \mathrm{X}_{2}, \mathrm{Y}_{2}\right)$ were defined to calculate the positions of the two masses $m_{1}$ and $m_{2}$ in the $x y$ plane.

Finally, the software evaluates these new parameters and the results are used to plot variation in position of pendulum bobs $\left(m_{1}\right.$ and $\left.m_{2}\right)$ with respect to time along the $x$ - and $y$-axis, respectively.

Due to space constraints, the Maple commands are not displayed here but can be viewed as supplementary files attached to this article. Only motions of the pendulum bobs $\left(m_{1}\right.$ and $\left.m_{2}\right)$ are displayed in Fig. 2 for $g=9.8, k_{1}=k_{2}=$ $0.01, L_{1}=L_{2}=0.5, m_{1}=m_{2}=0.1, q_{1}=q_{2}=1, \mu_{1}=\mu_{2}=0.05$.

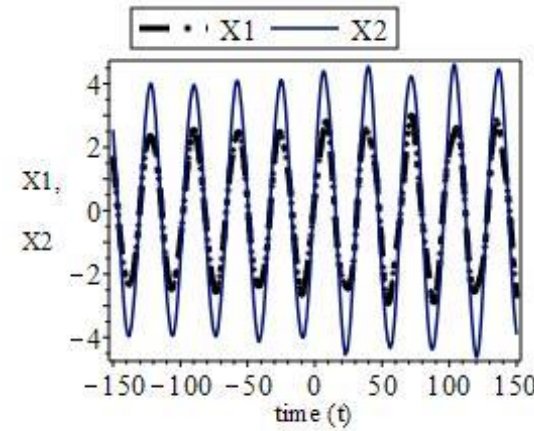

(a)

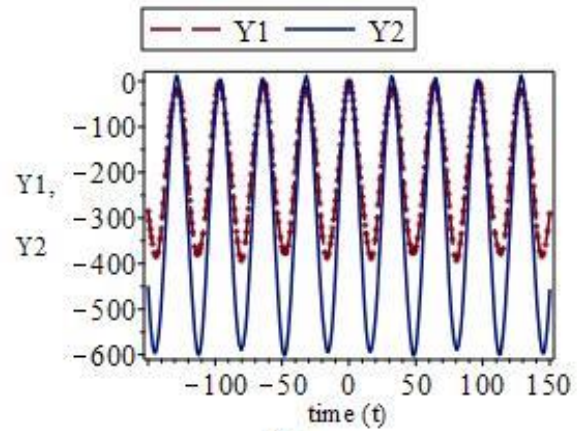

(b)

Fig. 2. Comparison between the motions of mass $m_{1}$ and $m_{2}$ along the (a) horizontal and (b) vertical planes.

Figure 2 represents variation in position of the pendulum bobs $m_{1}$ and $m_{2}$ with respect to time along the $x$ - and $y$-axis. The plots show sinusoidal curves that are continuous and periodic with slightly changing amplitudes. Figure 2(a) is characteristics of a sine wave function while Figure 2(b) is characteristics of a cosine wave function. The slightly varying amplitudes observed in the curves might be as a result of the chaotic nature of single and double spring-pendulum. Figure 2 shows comparison of the motion of pendulum bob $m_{1}$ and $m_{2}$. It is observed that the amplitudes of the curves representing motion for $m_{1}$ are smaller than those of motion for $m_{2}$ as displayed by Figures 2(a) and 2(b), respectively. 
Furthermore, we studied the behaviour of the pendulum bobs for: $k_{1} \neq k_{2}, q_{1} \neq q_{2}, m_{1} \neq m_{2}$, and $L_{1} \neq L_{2}$. For $k_{2}>k_{1}$, curves representing the motion of the pendulum bobs are shown in Figures 3(a) and (b). When $k_{1}$ is fixed at 0.01 , and the stiffness constant of the second spring $\left(k_{2}\right)$ is increased from 0.05 to 1 , we observed that the two bobs are in phase, periodic and the amplitudes are suppressed as displayed in Figures 3(a) and (b). This is an indication of a non-chaotic regime. Furthermore, the wave forms of the two bobs along the $x$-plane (X1, X2, X3, X4, X5 and X6) are seen to diverge as they progress with time.

(a)
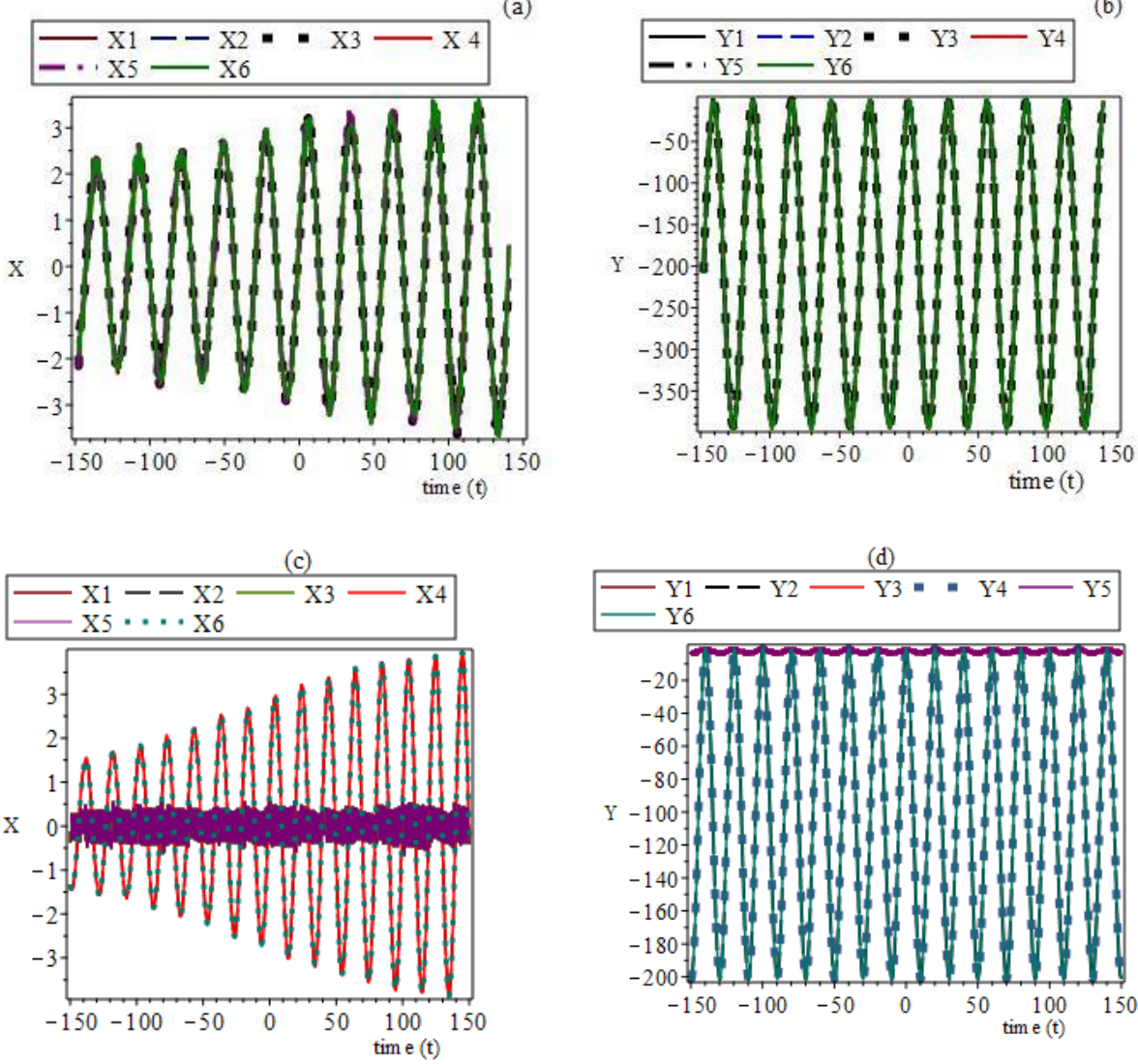

Fig. 3. Motion of inner and outer bob along the horizontal and vertical planes for $g=9.8, L_{1}=L_{2}=0.5, m_{1}=m_{2}=0.1, q_{1}=q_{2}=1$ and $\mu_{1}=\mu_{2}=0.05$ where (a) and (b) for $k_{2}>k_{1}$, and (c) and (d) for $k_{1}>k_{2}$.

In Figures 3(a \& b): X1, X2, Y1 and Y2 represent motions of pendulum bob (1\&2) along the $x$ - and $y$-plane for $k_{1}=0.01$ and $k_{2}=0.05 . \mathrm{X} 3, \mathrm{X} 4, \mathrm{Y} 3$ and Y4 represent motions of bobs along the $x$ - and $y$-plan for $k_{1}=0.01$ and $k_{2}=$ 0.5 , and X5, X6, Y5 and Y6 represent motions of bobs along the $x$ - and $y$ - 
plane for $k_{1}=0.01$ and $k_{2}=1$. In Figures 3(c\&d): X1, X2, Y1 and Y2 represent motions of pendulum bobs for $k_{1}=0.05$ and $k_{2}=0.01$. X3, X4, Y3 and $\mathrm{Y} 4$ represent motions of bobs for $k_{1}=0.5$ and $k_{2}=0.01$, and X5, X6, Y5 and Y6 represent motions of bobs for $k_{1}=1$ and $k_{2}=0.01$.
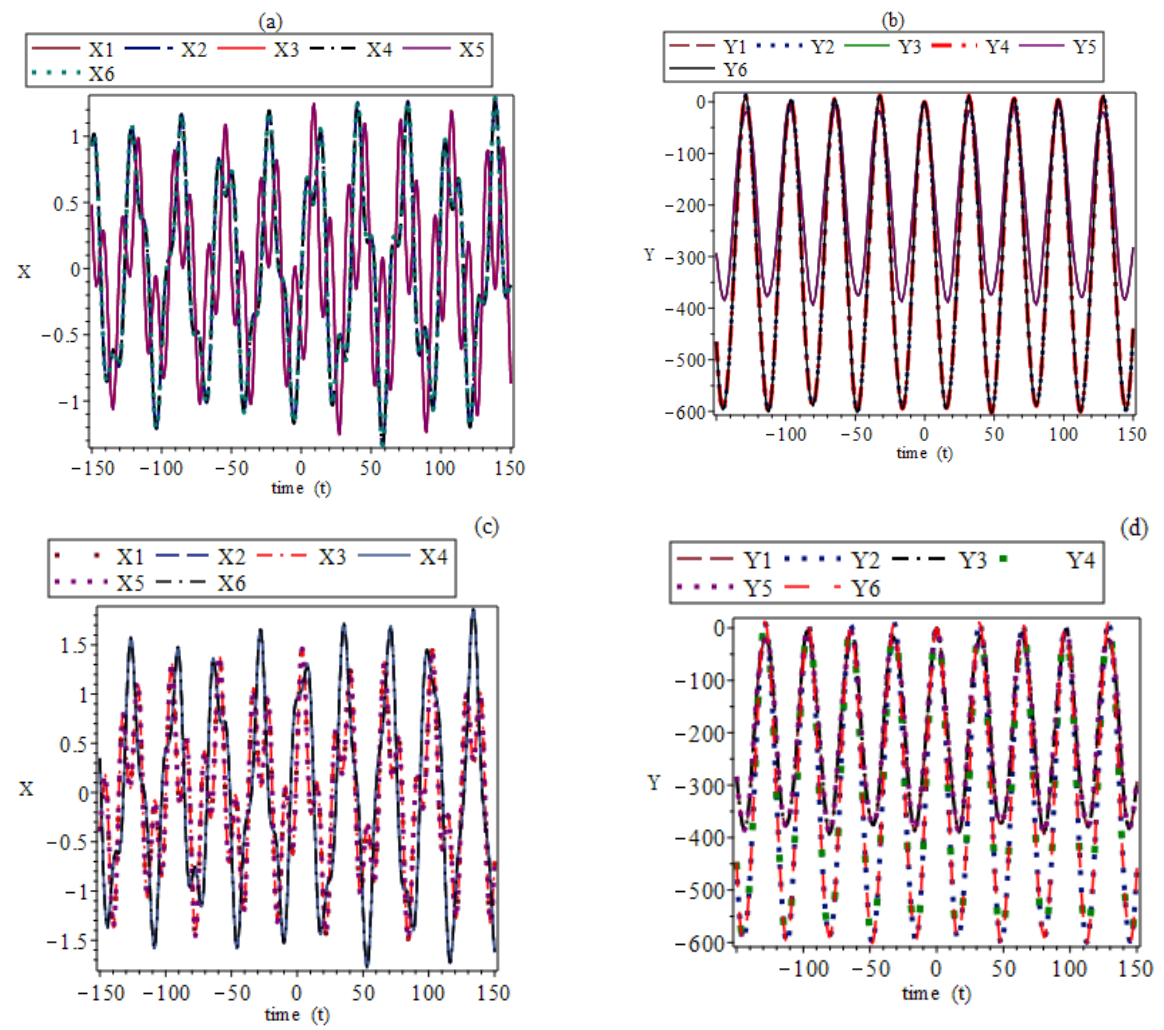

Fig. 4. Motion of the inner and outer bob along the horizontal and vertical planes for $g=9.8, L_{1}=L_{2}=0.5, m_{1}=m_{2}=0.1, k_{1}=k_{2}=0.01$ and $\mu_{1}=\mu_{2}=$ 0.05 where (a) and (b) for $q_{1}>q_{2}$, and (c) and (d) for $q_{2}>q_{1}$.

For $k_{1}>k_{2}$, the motions of the bobs are displayed in Figures 3(c \& d). As the stiffness constant of the first spring $k_{1}$ is increased from 0.05 to 1 with $k_{2}$ fixed at 0.01 , we entered a non-periodic regime. This can be likened to increase in energy that gives rise to quasi-periodic regime as reported by Biglari and Jami (2016). This is clearly seen in the curves associated with the motion of the inner bob $m_{1}$ along both $x$ and $y$ planes as shown in Figures 3(c \& d). However, we observed that for the curves representing the outer pendulum bob $m_{2}$ along the vertical plane are periodic with negative values. 
Also, keeping the angle of displacement $q_{2}$ fixed at 1 , and increasing $q_{1}$ $(=3,4.5,4.7)$, we observed a chaotic behaviour and as well the amplitudes are suppressed as shown in Figure 4(a). While for curves representing positions of the inner and outer bobs along the $y$ plane show only slight variation in amplitude (see Figure 4(b)).

In Figures 4(a \& b): X1, X2, Y1 and Y2 represent motions of pendulum bob (1\&2) along the $x$ - and $y$-plane for $q_{1}=3$ and $q_{2}=1$. X3, X4, Y3 and Y4 represent motions of bobs along the $x$ - and $y$-plane for $q_{1}=4.5$ and $q_{2}=1$, and X5, X6, Y5 and Y6 represent motions of bobs along the $x$ - and $y$-plane for $q_{1}=4.7$ and $q_{2}=1$. In (c \& d): X1, X2, Y1 and Y2 represent motions of pendulum bobs for $q_{1}=1$ and $q_{2}=0.5 . \mathrm{X} 3, \mathrm{X} 4, \mathrm{Y} 3$ and $\mathrm{Y} 4$ represent motions of bobs for $q_{1}=1$ and $q_{2}=1.5$, and X5, X6, Y5 and Y6 represent motions of bobs for $q_{1}=1$ and $q_{2}=2$.

Increasing $q_{2}(=0.5,1.5,2)$ with $q_{1}$ fixed, we noticed the two bobs do not move together, amplitudes of the graphs are suppressed and there exist quasiperiodic behaviour indicating a chaotic regime as displayed in Figure 4(c). In addition, when the mass of the outer pendulum bob $m_{2}$ is fixed at 0.05 and $m_{1}$ is increased from 0.1 to 2, we noticed from Figure 5(a) that the two bobs do not move together along the $x$ plane. Again this indicates the presence of chaos; as such X1, X2, X3, X4, X5 and X6 are out of phase. But, in Figure 5(b) the two bobs are in phase and periodic along the $y$ plane. Also, we observed that the amplitudes of the graphs have a tendency to increase with mass. On the other hand, when $m_{1}$ is fixed and $m_{2}$ is varied, one noticed from Figures ( $c \& d$ ), that the bobs move together along both axes. Nevertheless, there are indications of the presence of chaos on the graphs X1, X3, X5 and $\mathrm{X} 7$ as $m_{2}$ is increased from 0.1 to 2 .

In Figures 5 (a \& b): X1, X2, Y1 and Y2 represent motions of pendulum bob (1\&2) along the $x$ - and $y$-plane for $m_{1}=0.1$ and $m_{2}=0.05$. X3, X4, Y3 and Y4 represent motions of bobs along the $x$ - and $y$-plane for $m_{1}=0.5$ and $m_{2}=0.05$, and X5, X6, Y5 and Y6 represent motions of bobs along the $x$ - and $y$-plane for $m_{1}=1$ and $m_{2}=0.05 . \mathrm{X} 7, \mathrm{X} 8, \mathrm{Y} 7$ and Y8 represent curves for bobs along the $x$ - and $y$-plane for $m_{1}=2$ and $m_{2}=0.05$. In (c \& d): X1, X2, Y1 and Y2 represent curves for pendulum bobs along the $x$ - and $y$-plane for $m_{1}=0.05$ and $m_{2}=0.1 . \mathrm{X} 3, \mathrm{X} 4, \mathrm{Y} 3$ and $\mathrm{Y} 4$ represent curves for bobs along the $x$ - and $y$-plane for $m_{1}=0.05$ and $m_{2}=0.5$. X5, $\mathrm{X} 6$, Y5 and Y6 represent curves for bobs along the $x$ - and $y$-plane for $m_{1}=0.05$ and $m_{2}=1$, and $\mathrm{X} 7, \mathrm{X} 8, \mathrm{Y} 7$ and $\mathrm{Y} 8$ represent curves for bobs along the $x$ - and $y$-plane for $m_{1}=0.05$ and $m_{2}=2$. 

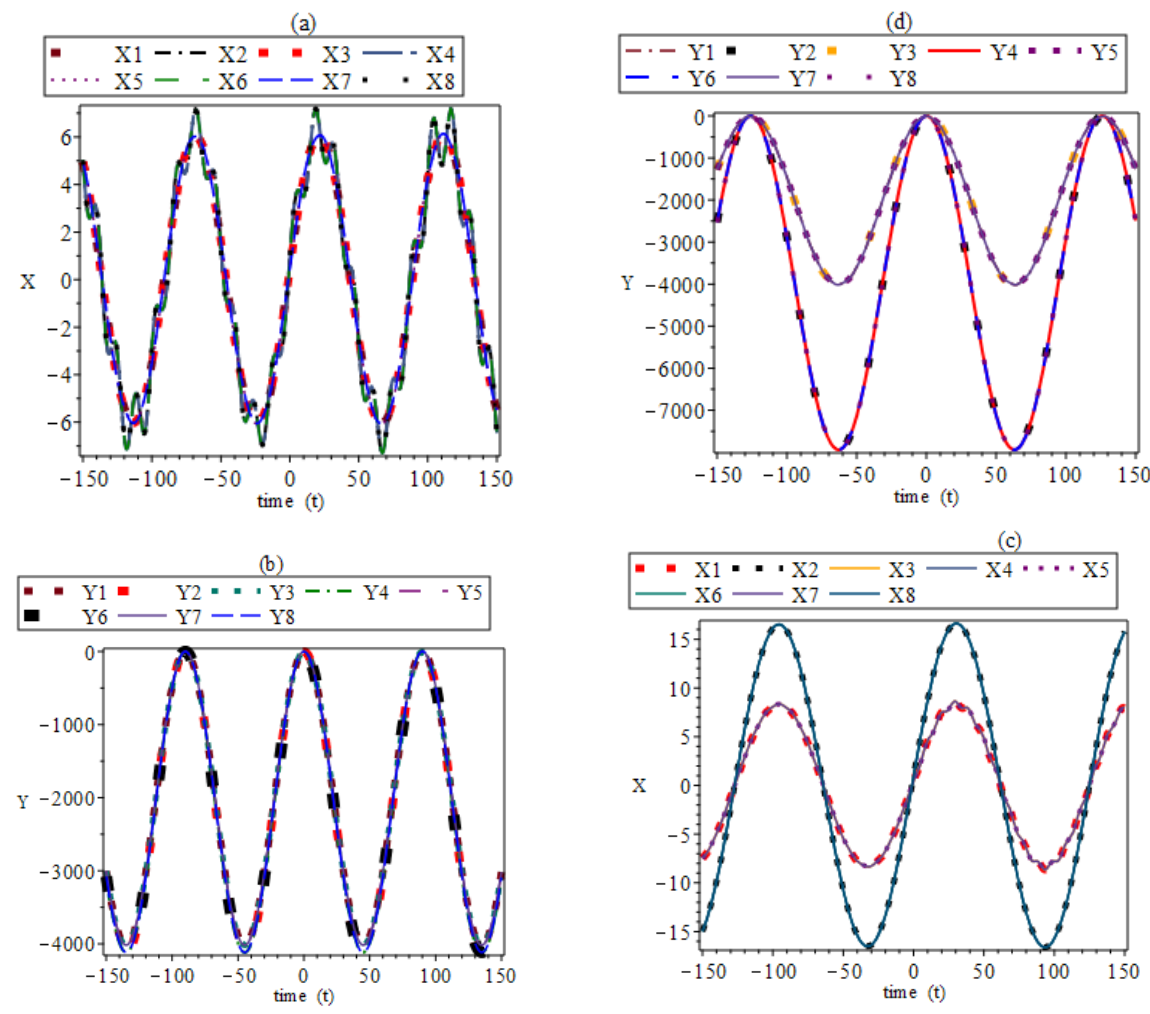

Fig. 5. Motion of the inner and outer bob along the horizontal and vertical planes for $g=9.8, L_{1}=L_{2}=0.5, q_{1}=q_{2}=1, k_{1}=k_{2}=0.01$ and $\mu_{1}=\mu_{2}=0.05$ where (a) and (b) for $m_{1}>m_{2}$, and (c) and (d) for $m_{2}>m_{1}$.

Finally, increasing the length of the inner or outer spring has significant effect on the motion of the pendulum bobs. Keeping $L_{1}$ fixed and increasing the length of the outer spring $L_{2}$, or keeping $L_{2}$ fixed and increasing the length of the inner spring $L_{1}$, we observed that the inner and outer pendulum bobs do not move together along the $x$ plane as shown in Figures $6(\mathrm{a} \& \mathrm{c})$. Hence, signifies the existence of a chaotic regime. On the other hand, along the $y$ plane the two bobs are in phase and periodic showing there is no chaos along this plane. This periodic and chaotic behaviour is consistent with previous studies on spring-pendulum systems (Lewin and Chen 2015, CarreteroGonzalez et al. 1994, Leah 2013, Nunez-Yepez et al. 1990). 

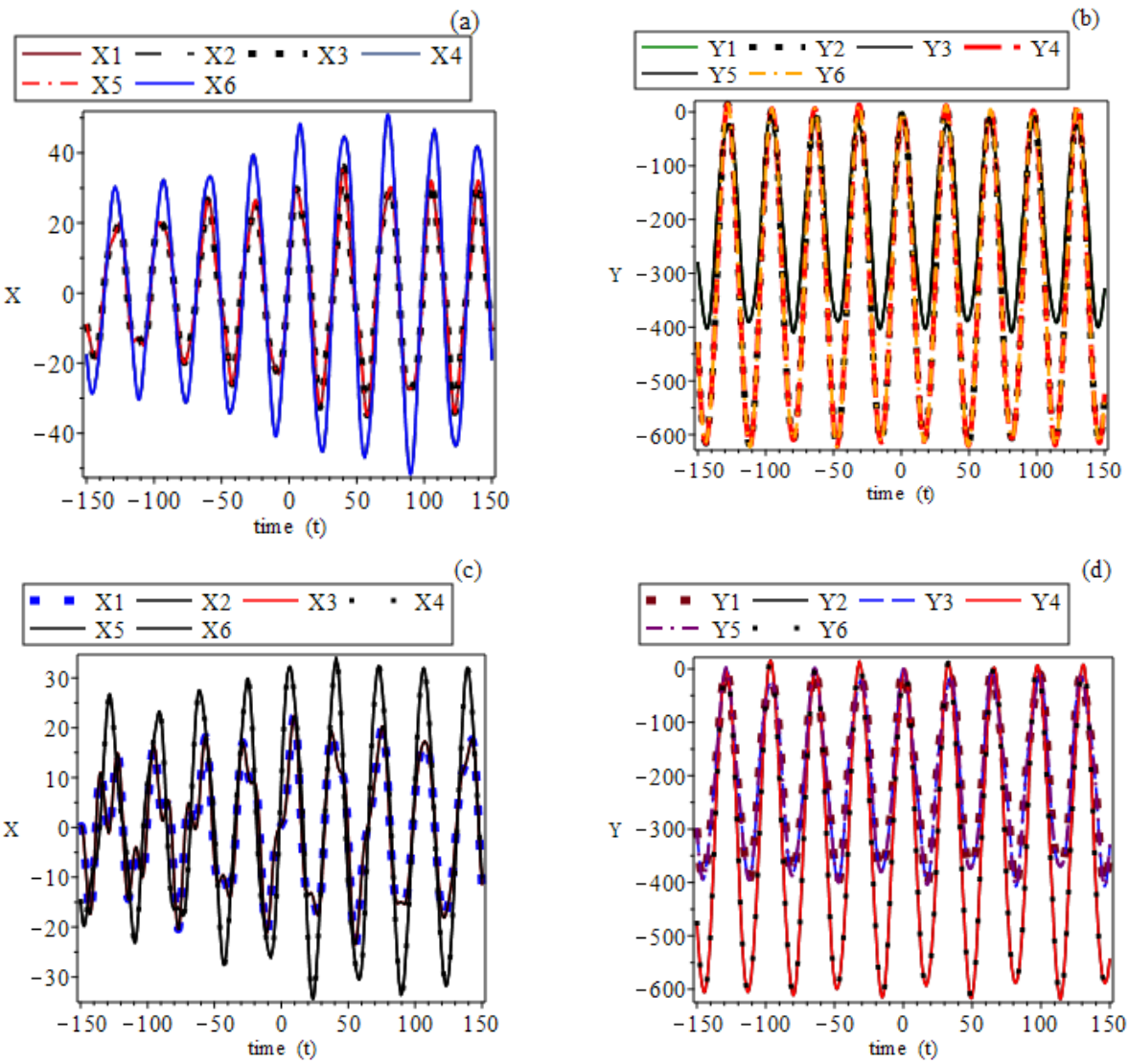

Fig. 6. Motion of the inner and outer bob along the horizontal and vertical planes for $g=9.8, m_{1}=m_{2}=0.1, q_{1}=q_{2}=1, k_{1}=k_{2}=0.01$ and $\mu_{1}=\mu_{2}=0.05$ where (a) and (b) $L_{1}>L_{2}$, , and (c) and (d) $L_{2}>L_{1}$.

In Figures 6 (a \& b): X1, X2, Y1 and Y2 represent motions of pendulum bobs (1\&2) along the $x$ - and $y$-plane for $L_{1}=2$ and $L_{2}=0.5 . \mathrm{X} 3, \mathrm{X} 4, \mathrm{Y} 3$ and Y4 represent motions of bobs along the $x$ - and $y$-plane for $L_{1}=5$ and $L_{2}=0.5$, and X5, X6, Y5 and Y6 represent motions of bobs along the $x$ - and $y$-plane for $L_{1}=10$ and $L_{2}=0.5$. In (c \& d): X1, X2, Y1 and Y2 represent motions of pendulum bobs for $L_{1}=0.5$ and $L_{2}=2$. X3, X4, Y3 and Y4 represent motions of bobs for $L_{1}=0.5$ and $L_{2}=5$, and X5, X6, Y5 and Y6 represent motions of bobs for $L_{1}=0.5$ and $L_{2}=10$. 


\section{Conclusions}

Equations of motion for the 2D double spring-pendulum with time dependent spring extension are derived using Lagrangian formulation of mechanics in generalized coordinates and are solved approximately. These equations were also solved numerically with Maple and it was observed that motion along the $\mathrm{x}$-axis is characterized by a sine wave curve while motion along the $\mathrm{y}$-axis is characterized by a cosine wave curve. Change in stiffness constant, angle of deflection, mass of pendulum bob and spring length were found to have significant effect on the dynamics of a $2 \mathrm{D}$ double spring-pendulum. The periodic and chaotic behaviour noticed in this study is consistent with current literature on spring-pendulum systems.

\section{Acknowledgements}

Comments from two anonymous RJS reviewers on the initial draft of the submitted manuscript are acknowledged.

\section{References}

Baleanu D, Asad, JH, Petras I. 2015. Numerical solution of the fractional EulerLagrange's equations of a thin elastic model. Nonlinear Dynamics 81: 97-102. https://doi.org/10.1007/s11071-015-1975-7

Biglari H, Jami RA. 2016. The double pendulum numerical analysis with Lagrangian and the Hamiltonian equations of motions. Conference paper: International conference on mechanical and aerospace engineering. pp 1-12. https://www.researchgate.net/publication/ 301890703

Carretero-Gonzalez R, Nunez-Yepez NH, Salas-Brito LA. 1994. Regular and chaotic behaviour in an extensible pendulum, European Journal of Physics 15: 139. https://doi.org/10.1088/ 0143-0807/15/3/009

de Sousa MC, Marcus FA, Caldas IL. 2017. Energy distribution in spring pendulum. Nonlinear Optics 4: 6 .

Goldstein H, Poole C, Safko J. 2000. Classical mechanics, $3^{\text {rd }}$ edn. Addison Wesley, New York, 5-69.

Leah G. 2013. The Swinging Spring: Regular and Chaotic Motion. http://depts.washington.edu/ amath/wordpress/wp-content/uploads/2014/01/leah_ganis_pres.pdf

Lewin A, Chen P. 2015. Numerical analysis of dynamics of single and double spring pendulum systems. https://www.math.dartmouth.edu/ m53f15/proj/chenlewin.pdf

Maple 2018. Maplesoft, a division of Waterloo Maple Inc., Waterloo, Ontario.

Marcus FA, de Sousa MC, Caldas IL. 2016. Order-chaos-order transition in a spring pendulum, $6^{\text {th }}$ International Conference on Nonlinear and Complexity, National Institute for Space Research, Brazil, May 16-20.

Martin C, Salomonson P. 2009. An introduction to analytical mechanics, $4^{\text {th }}$ edn. Goteborg Sweden 1-62 pp.

Murray RS. 1967. Theoretical mechanics, Schaum's outlines, Mcgraw-Hill 299 - 301 pp.

Nunez-Yepez NH, Salas-Brito LA,Vicente L. 1990. Onset of Chaos in an Extensible Pendulum. Physics Letters A 145:101-105. https://doi.org/10.1016/0375-9601(90)90199-X

Smith RA. 2002. Numerical solution of equations of motion for double pendulum, Maple document Application center.1-5 pp. 